\title{
Emergent Percutaneous Cardiopulmonary Bypass in Patients Having Cardiovascular Collapse in the Cardiac Catheterization Laboratory
}

\author{
David W. Grambow, MD, G. Michael Deeb, MD, Gregory S. Pavlides, MD, Ann Margulis, RN, \\ William W. O'Neill, MD, and Eric R. Bates, MD
}

\begin{abstract}
Percutanoous cardiopulmonary bypass (PCB) was instituted in 30 initially stable patients who developed either cardiac arrest refractory to resuscltation $(n=7)$ or cardiogenic shock (mean arterial blood pressure $<50 \mathrm{~mm} \mathrm{Hg}$ unresponsive to fluld resuscitation or vasopressors) $(n=23)$ after a catheterization laboratory complication. Events leading to collapse included abrupt closure during percutaneous transluminal coronary angioplasty (PTCA) $(n=22)$, complications from diagnostic cardiac catheterization $(n=6)$, left ventricular perforation during mitral valvuloplasty $(n=1)$, and right ventricular perforation during poricardiocentosis $(n=1)$. PCB was initiated within 20 minutes of cardiovascular collapse in 83\% of patients (arrest: $21 \pm 13$ minutes [range 10 to 50]; and shock: $17 \pm 6$ minutes [range 10 to 30]). Mean arterial blood pressure increased on PCB from 0 to $56 \mathrm{~mm} \mathrm{Hg}$ in patients with cardiac arrest and from 37 to $63 \mathrm{~mm}$ Hg in those with cardiogenic shock at mean PCB flow rates of 2.5 to 5.0 liters/min. Subsequent therapy on PCB included emergent cardiac surgery ( $n=14)$, PTCA $(n=13)$ and modical therapy $(n=3)$. Six patients $(20 \%)$ survived to hospital discharge ( 3 with cardlac surgery, 2 with PTCA, and 1 with medical therapy). All 7 patients with refractory cardiac arrest dled despite further interventions on PCB, whereas 6 of 23 (26\%) with cardiogenic shock survived to hospital discharge. Thus, in response to cardiovascular collapse in the catheterization laboratory, PCB does not salvage patients who do not regain a stable cardiac rhythm. PCB can stabilize patients who develop cardiogenic shock for further Interventions which are lifesaving in only a minority of pationts.
\end{abstract}

(Am J Cardiol 1994;73:872-875)

From the Cardiology Division, Department of Internal Medicine, and Department of Thoracic Surgery, University of Michigan, Ann Arbor, Michigan; and the Department of Cardiology, William Beaumont Hospital, Royal Oak, Michigan. Manuscript received July 7, 1993; revised manuscript received and accepted October 7, 1993.

Address for reprints: Eric R. Bates, MD, Division of Cardiology, B1-F245, University Hospital, University of Michigan, 1500 East Medical Center Drive, Ann Arbor, Michigan 48109-0022.
Dercutaneous cardiopulmonary bypass (PCB) has been used in the cardiac catheterization laboratory to provide prophylactic support during percutaneous transluminal coronary angioplasty (PTCA) in patients with severe left ventricular dysfunction (left ventricular ejection fraction $<25 \%$ ) or during PTCA of a target vessel supplying $>50 \%$ of the remaining viable myocardium. ${ }^{1,2}$ PCB has also been used as a temporary emergency support measure in patients sustaining cardiovascular collapse from acute myocardial infarction, pulmonary embolism, trauma, drug overdose, unsuccessful PTCA, postoperative deterioration and aortic stenosis, and after rupture of aortic dissection. ${ }^{3-6}$ Two previous small studies suggested that the use of PCB in patients with cardiac arrest improved resuscitative efforts. ${ }^{4,6}$ However, the mechanism for this improvement is not clear, because direct myocardial perfusion does not occur while patients are on PCB, and regional myocardial ischemia has been shown when PCB is used in those with coronary artery disease. ${ }^{7}$ Therefore, we reviewed our experience with emergent PCB in patients developing refractory cardiac arrest or cardiogenic shock in the cardiac catheterization laboratory from a procedural related complication to further examine the use of this technology.

\section{METHODS}

Study pationts: A retrospective review was performed of the use of emergent PCB for cardiovascular collapse occurring in the cardiac catheterization laboratories of the University of Michigan and William Beaumont Hospitals. Between January 1988 and January 1992, 30 patients were identified who presented to the cardiac catheterization laboratory with stable hemodynamics and had PCB initiated emergently for either refractory cardiac arrest or cardiogenic shock occurring as a result of a catheterization laboratory complication. $\mathrm{Pa}$ tients were considered to have refractory cardiac arrest only if aggressive attempts at resuscitation were unsuccessful in establishing a stable cardiac rhythm. Patients were placed on PCB for cardiogenic shock if hypotension and peripheral hypoperfusion persisted despite aggressive fluid replacement and inotropic support.

Technique of percurtaneous cardiopulmonary bypass: A portable PCB system (C.R. Bard, Inc., Billerica, Massachusetts) was used, and included a centrifugal nonocclusive pump with a hollow-fiber membrane oxygenator and water-based heat exchanger. Femoral arterial and venous access were obtained contralateral to the site of cardiac catheterization. Over a 0.035 -inch guidewire, progressive dilatation of the femoral artery 


\begin{tabular}{|lccc|}
\hline TABLE I Clinical Characteristics & & \\
\cline { 1 - 2 } & & $\begin{array}{c}\text { Refractory } \\
\text { Cardiac Arrest }\end{array}$ & $\begin{array}{c}\text { Cardiogenic } \\
\text { Shock }\end{array}$ \\
\cline { 1 - 2 } & & 23 \\
Patients & $37 \pm 11$ & $63 \pm 6$ \\
Men (years) & $3(43 \%)$ & $14(61 \%)$ \\
Risk factors & & \\
Hypertension & $6(86 \%)$ & $16(70 \%)$ \\
Diabetes & $2(29 \%)$ & $3(13 \%)$ \\
Smoking & $1(15 \%)$ & $6(26 \%)$ \\
Hypercholesterolemia & $1(15 \%)$ & $7(34 \%)$ \\
Previous events & & \\
Myocardial infarction & $4(57 \%)$ & $10(44 \%)$ \\
Coronary angioplasty & $1(15 \%)$ & $3(13 \%)$ \\
Coronary bypass surgery & $1(15 \%)$ & $5(22 \%)$ \\
Angina class 3-4 & $5(72 \%)$ & $20(87 \%)$ \\
Congestive heart tailure class $3-4$ & $2(29 \%)$ & $3(13 \%)$ \\
Left ventricular ejection fraction (\%) & $46 \pm 20$ & $43 \pm 14$ \\
Number of arteries narrowed $>50 \%$ & & \\
in diameter & & \\
0 & $1(15 \%)$ & $1(5 \%)$ \\
1 & $2(29 \%)$ & $5(22 \%)$ \\
2 & $1(15 \%)$ & $7(30 \%)$ \\
3 & $3(42 \%)$ & $10(43 \%)$ \\
Left main & 0 & $6(23 \%)$ \\
\hline
\end{tabular}

was performed before insertion of an $18 \mathrm{Fr}$ cannula. An 18 Fr multihole femoral venous cannula was inserted by a similar technique, and was positioned with fluoroscopy at the junction of the inferior vena cava and right atrium. Intravenous heparin was administered to maintain an activated clotting time of $>400$ seconds throughout the procedure. The cannula system was connected to the portable PCB in a manner to ensure an air-free system. PCB was initiated at 2 liters of flow/min and was increased until the mean arterial blood pressure was $>60$ $\mathrm{mm} \mathrm{Hg}$ or further increases in flow were limited by inadequate venous return. The pulmonary capillary wedge pressure was maintained at $>10 \mathrm{~mm} \mathrm{Hg}$ to ensure unimpeded venous return at higher flow rates. Periodic monitoring of arterial blood gases, pulmonary capillary wedge pressures, electrocardiograms, coagulation variables, electrolytes and mixed venous oxygen saturations was performed.

Termination of percutaneous cardiopulmonary bypass: Patients achieving hemodynamic stability in the catheterization laboratory had PCB gradually weaned over 10 to 15 minutes, with an intraaortic balloon pump and inotropic agent support used as necessary. These patients were then sent for surgical removal of the cannula system. Patients having cardiac surgery were transported to the operating room on PCB and had PCB terminated after standard cardiopulmonary bypass was established.

\section{RESULTS}

Patient characteristics: Clinical and angiographic characteristics of patients are listed in Table I. Seven patients were placed on PCB because of refractory cardiac arrest and 23 because of cardiogenic shock. Of patients in cardiogenic shock, 16 (70\%) initially had cardiac arrest (mean duration 13 minutes [range 3 to 45]) and were successfully resuscitated before the initiation of PCB.

Events leading to cardlovascular collapse: Abrupt closure during PTCA was the most frequent cause of

\begin{tabular}{|c|c|c|c|}
\hline & $\begin{array}{c}\text { Refractory } \\
\text { Cardiac } \\
\text { Arrest }(n=7)\end{array}$ & $\begin{array}{l}\text { Cardiogenic } \\
\text { Shock } \\
(n=23)\end{array}$ & $\begin{array}{c}p \\
\text { Value }\end{array}$ \\
\hline Time to initiation of bypass (min) & $21 \pm 13$ & $17 \pm 6$ & NS \\
\hline Duration of bypass ( $\mathrm{min}$ ) & $40 \pm 22$ & $165 \pm 213$ & 0.2 \\
\hline $\begin{array}{l}\text { Mean arterial blood pressure on } \\
\text { bypass }(\mathrm{mm} \mathrm{Hg})\end{array}$ & $56 \pm 7$ & $63 \pm 9$ & NS \\
\hline
\end{tabular}

cardiovascular collapse needing PCB support $(n=22)$. Severe left main coronary artery disease was present in 5 patients needing PCB because of complications from diagnostic cardiac catheterization, whereas an additional one with 3-vessel coronary artery disease developed refractory cardiac arrest after contrast injection of the right coronary artery and was placed on PCB. Left ventricular perforation during mitral valvuloplasty, and right ventricular laceration with cardiac tamponade at pericardiocentesis were other catheterization laboratory complications for which emergent PCB was instituted.

Percutaneous cardiopulmonary support: PCB was initiated within 20 minutes of cardiovascular collapse in 25 of 30 patients $(83 \%)$ (Table II). The mean arterial blood pressure for patients in refractory cardiac arrest on PCB increased from 0 to $56 \mathrm{~mm} \mathrm{Hg}$ at flow rates of 3 to 5 liters/min. No patient in the refractory cardiac arrest group regained consciousness while on PCB. Mean arterial blood pressure in patients in cardiogenic shock on PCB increased from 37 to $63 \mathrm{~mm} \mathrm{Hg}$ at flow rates of 2.5 to 5.0 liters $/ \mathrm{min}$.

Treatment after initiation of percutaneous cardiopulmonary bypass: After PCB was established, 14 patients were sent for emergent cardiac surgery, 13 had repeat PTCA, and 3 were not considered candidates for revascularization and were managed medically. Six patients (20\%) survived to hospital discharge. All $7 \mathrm{pa}-$ tients with refractory cardiac arrest died despite further interventions on PCB. Emergent cardiac surgery was performed in 3 patients ( 2 coronary artery bypass graft procedures and 1 repair of right ventricular laceration); however, none were able to be weaned from PCB after surgery. Repeat PTCA was performed on PCB in 4 patients, but none regained a stable cardiac rhythm after PTCA and all died in the cardiac catheterization laboratory.

Of patients having PCB for cardiogenic shock, 13 of $23(57 \%)$ survived 24 hours after the initial intervention on PCB and were successfully weaned from PCB, but only $6(26 \%)$ were discharged eventually from the hospital. Subsequent deaths in early surviving patients were due to refractory congestive heart failure $(n=4)$, sepsis $(n=2)$ and multisystem failure $(n=1)$. The surgical interventions in patients with cardiogenic shock included coronary artery bypass grafting $(n=9)$, repair of left ventricular perforation $(n=1)$, and orthotopic cardiac transplantation $(\mathrm{n}=1)$ after 2 days on an extracorporeal membrane oxygenation system. Surgical patients surviving to hospital discharge included 2 who underwent coronary artery bypass grafting, and 1 who had cardiac transplantation. Repeat PTCA on PCB was performed 


\begin{tabular}{|c|c|c|c|}
\hline Study & $\begin{array}{l}\text { Number } \\
\text { of Patients }\end{array}$ & $\begin{array}{l}\text { Early Surviving } \\
\text { Patients }\end{array}$ & Discharged \\
\hline Baird et al 12 & 19 & 3 & $3(16 \%)$ \\
\hline Mattox and Beall14 & 43 & & $17(40 \%)$ \\
\hline Phillips et al ${ }^{13}$ & 5 & & $3(60 \%)$ \\
\hline Reichman et al ${ }^{3}$ & 36 & 18 & $6(17 \%)$ \\
\hline Shawl et al ${ }^{4}$ & 7 & 6 & $4(57 \%)$ \\
\hline Mooney et al 6 & 11 & & $7(64 \%)$ \\
\hline Present study & 7 & 0 & 0 \\
\hline Total & 128 & & $40(31 \%)$ \\
\hline
\end{tabular}

in 9 patients. PTCA was successful in 7 patients; 6 were weaned from PCB before leaving the catheterization laboratory, with 2 eventually surviving to hospital discharge. Three patients with cardiogenic shock were not considered candidates for surgery or PTCA; intraaortic balloon counterpulsation with inotropic agent support enabled 2 of these to be weaned from PCB, and 1 survived to hospital discharge.

Complications: Most PCB-related complications were related to cannula insertion sites. Bleeding needing transfusion occurred in 8 patients. A femoral artery thrombosis developed in 1 patient after sheath removal, and 2 needed surgical repair of femoral artery fistulae.

Follow-up: The mean hospital stay in the 6 patients surviving to discharge was 22 days (range 6 to 62 ). Follow-up was available in 5 patients at a mean of 18 weeks. Four patients were free of cardiac symptoms, and 1 was being managed medically for recurrent stable angina.

\section{DISCUSSION}

Percutaneous cardiopulmonary bypass was advocated recently for prophylactic use in high-risk PTCA $^{1}$ and for emergency use in sudden cardiovascular collapse. ${ }^{2-6}$ In contrast to the intraaortic balloon pump, PCB can maintain hemodynamic stability in the absence of an intrinsic cardiac rhythm or effective cardiac output and can improve tissue perfusion in cases of severe left ventricular failure; it cannot be used in patients with ileofemoral disease nor for $>24$ hours, because of disruption of blood elements.

The rationale for use of PCB in patients with cardiovascular collapse is to provide temporary hemodynamic support for subsequent percutaneous or surgical intervention. Rapid initiation of PCB within 20 minutes of cardiovascular collapse appears necessary if subsequent interventions are to prove successful in achieving hemodynamic stability. ${ }^{1}$ The ability to rapidly place a patient on PCB in the catheterization laboratory suggests that PCB could help salvage a large number of patients with cardiovascular collapse from catheterization laboratory complications. However, several studies suggest that whereas PCB supports the peripheral circulation, coronary blood flow is not maintained and actually decreases when PCB is initiated. ${ }^{7}$ This decrease in coronary blood flow has been hypothesized to be a result of coronary steal ${ }^{8}$ or impaired autoregulation of the cardiac microcirculation from nonpulsatile flow. ${ }^{9}$ Pavlides et $\mathrm{al}^{7}$ have shown that regional and global left ventricular dysfunction occur with PTCA balloon inflation during PCB despite reductions in measured afterload and maximal oxygen consumption requirements. Myocardial regions supplied by stenotic vessels had deterioration of wall motion when patients were placed on PCB before balloon inflation and had further deterioration with balloon inflation. Thus, whereas cerebral, renal and hepatic blood flow are maintained on PCB during cardiovascular collapse, coronary blood flow is not equally preserved, and progressive myocardial ischemia occurs in patients with coronary artery disease. Resuscitation from refractory cardiac arrest on PCB would be more difficult owing to this progressive ischemia.

We found PCB to be useful in stabilizing patients in cardiogenic shock as a result of a catheterization laboratory complication, including patients initially presenting with cardiac arrest who were successfully resuscitated to a stable rhythm before PCB initiation. However, we did not find use for this technique in patients with refractory cardiac arrest. No patient with refractory cardiac arrest in this study was salvaged with the use of PCB, although 6 of 7 were placed on PCB within 20 minutes of arrest.

Early studies of animals suggested that cardiopulmonary bypass could successfully facilitate defibrillation in dogs with intractable ventricular fibrillation ${ }^{10,11}$; however, its usefulness in refractory cardiac arrest in humans is controversial. $3,4,6,12,13$ Table III reviews the results of studies that evaluated the use of emergent PCB in cardiac arrest. Survival rates ranged from 16 to $64 \%$. Events preceding cardiac arrest and location within the hospital when PCB was initiated widely varied between studies. In the study by Baird et al, ${ }^{12}$ PCB was begun for cardiac arrest in patients presenting to the hospital with acute myocardial infarction. In other studies, PCB was initiated for cardiac arrest occurring as a complication of several medical conditions including trauma, sepsis, drug overdose, pulmonary embolism, myocardial infarction, cardiomyopathy and postoperative collapse. ${ }^{3,13,14}$ PCB was initiated in many different hospital locations ranging from the emergency room to the intensive care unit. Many of the aforementioned studies did not specify the specific ctiology of the cardiac arrest, the duration of the arrest nor the extent of the resuscitative efforts performed before initiation of PCB.

Shawl et $\mathrm{al}^{4}$ and Mooney et $\mathrm{al}^{6}$ were the first to examine PCB for cardiac arrest occurring in, or in proximity to, the catheterization laboratory and reported the highest late survival rates for this technique. However, the extent of resuscitative efforts before initiating PCB was not discussed. Previous studies reporting a high success of PCB in reversing refractory cardiac arrest may have included patients who would have regained stable cardiac rhythm with more persistent resuscitation alone. In the present study, 23 of 30 patients placed on PCB had cardiac arrest, but 16 of these regained a stable cardiac rhythm during resuscitation before initiation of PCB. Thirty percent of the resuscitated patients survived to hospital discharge, while none of the 7 with refractory cardiac arrest survived despite PCB. 
Study limitations: The retrospective nature of this investigation, together with the small study size, limits generalization of the findings. All patients developed cardiovascular collapse after a cardiac catheterization laboratory complication, and thus the use of PCB in cardiovascular collapse from other etiologies cannot be extrapolated.

1. Vogel RA, Shawl F, Tommaso C, O'Neill W, Overlie P, O'Toole J, Vandormael M, Topol E, Tabari KK, Vogel J, Smith S, Freedmann R, White C, George $B$, Teirstein P. Initial report of the National Registry of Elective Cardiopulmonary Bypass Supported Coronary Angioplasty. J Am Coll Cardiol 1990;15:23-29.

2. Shawl F, Domanski M, Punja S, Hemandez TJ. Percutaneous cardiopulmonary bypass support in high-risk patients undergoing percutaneous transluminal coronary angioplasty. Am J Cardiol 1989;64:1258-1263.

3. Reichman RT, Joyo CI, Dembitsky WP, Griffith LD, Admonson RM, Daily PO, Uverlie PA, Smith SC, Jaski BE. Improved patient survival after cardiac arrest using a cardiopulmonary support system. Ann Thorac Surg 1990;49:101-105.

4. Shawl FA, Domanski MJ, Wish MH, Davis M, Punja S, Herandez TJ. Emergency cardiopulmonary bypass support in patients with cardiac arrest in the catheterization laboratory. Cathet Cardiovasc Diag 1990;19:8-12.

5. Shawl FA, Domanski MJ, Hemandez TJ, Punja S. Emergency percutaneous cardiopulmonary bypass support in cardiogenic shock from acute myocardial infarction. Am J Cardiol 1989;64:967-970.
6. Mooney MR, Arom KV, Joyce LD, Mooney JF, Goldenberg IF, Von Rueden TJ, Emery RW. Emergency cardiopulmonary bypass support in patients with cardiac arrest. J Thorac Cardiovasc Surg 1991;101:450-454.

7. Pavlides GS, Hauser AM, Stack RK, Dudlets PI, Grines C, Timmis GC, O'Neil WW. Effect of peripheral cardiopulmonary bypass on left ventricular size, afterload and myocardial function during elective supponed coronary angioplasty. $\mathrm{J} \mathrm{Am}$ Coll Cardiol 1991;18:499-505.

8. Ciardullo RC, Schatt HV, Flaherty JT. Comparison of regional myocardial blood flow and metabolism distal to a critical coronary stenosis in the fibrillating hear during alternate periods of pulsatile and non-pulsatile perfusion. $J$ Thorac Cardiovasc Surg 1978;75:193-204

9. Mooney MR, Jishman-Mooney J, Mathias D. Clinical applications of percutaneous cardiopulmonary bypass for high risk coronary angioplasty. $J$ Invest $C$ ardiol 1990;2:161-169.

10. Proctor E, Kowalik TA. Circulatory support by pump-oxygenator in experimental ventricular fibrillation and acute left heart failure induced by coronary artery ligation. Cardiovasc Res 1967;1:189-193.

11. Evans D, Miyagishima R'I, Tutassaura H, Ameli M, Baird RJ. The effects of closed chest venoarterial bypass with oxygenation on cardiopulmonary hemodynamics. $J$ Thorac Cardiovasc Surg 1971;1:76-83.

12. Baird RJ, de la Rocha AG, Miyagishima K'I', 'Tutassaura $H$, Wilson DR, Evans D. Beanlands DS. Assisted circulation following myocardial infarction: a review of 25 patients treated before 1971. Can Med Assoc J 1972;107:287-291.

13. Phillips SJ, Ballentine B, Slonine D, Hall J, Vandehaar J, Kongtahworn C. Reckmo K, Gray D. Percutaneous initiation of cardiopulmonary bypass. Ann Thorac Surg 1983;36:223-225.

14. Mattox KL, Beall AC. Application of portable cardiopulmonary bypass. J Am Coll Emerg Physicians 1975;4:528-531. 